\title{
A dynamic scattering approach for a gated interacting wire
}

\author{
Inès Safi \\ Service de Physique de l'État Condensé, Centre d'Études de Saclay \\ 91191 Gif-sur-Yvette, France
}

\begin{abstract}
A new scattering approach for correlated one-dimensional systems is developed. The adiabatic contact to charge reservoirs is encoded in time-dependent boundary conditions. The conductance matrix for an arbitrary gated wire, respecting charge conservation, is expressed through a dynamic scattering matrix. It is shown that the dc conductance is equal to $e^{2} / h$ for any model with conserved total left- and right-moving charges. The ac conductance matrix is explicitly computated for the interacting Tomonaga ${ }_{2}$ Luttinger model (TLL).

Pioneered by Landauer, the scattering approach for quantum transport has proven powerful in mesoscopic physics. Nevertheless, it is restricted to non-interacting systems, and to the stationary regime. There were formal extensions to finite frequency transport based on a self-consistent approach, 0 or non-equilibrium techniques for interacting dots 3 but these formalisms are difficult to exploit. Proposed here is a new scattering approach at arbitrary frequency for linear transport through a strongly correlated, one-dimensional wire in the low-
\end{abstract} energy regime. Charge reservoirs connected adiabatically to the wire are accounted for by appropriate boundary conditions. Coupling to a gate is taken into account, ensuring charge conservation. The corresponding AC $3 \times 3$ conductance matrix is expressed through a novel dynamic "scattering" matrix $\mathbf{S}(\omega)$. Further progress is then made in two cases. First, for any model where the total charge for right- and left-moving electrons is conserved, the transmission is shown to be unity in the zero-frequency limit. This generalizes the DC conductance result $\AA \overline{\bar{n}} e^{2} / h$ shown for a Tomonaga-Luttinger liquid (TLL) 6 or arbitrary finite-range interactions. The same result was shown in Ref. [8] through different arguments restricted to the stationary regime, without describing the reservoir-wire interface. Second, $\mathbf{S}(\omega)$ is computed for the TLL model, giving an AC conductance that depends on interactions in contrast to the stationary regime.

Without connecting one-dimensional leads to an interacting wire, this work extends the concept introduced in Refs. [ 4, 9, 10] where reservoirs are simulated by the electrons they inject. The leads have served to define the incident and transmitted electrons, different from the proper modes of the wire. $\mathbf{S}(\omega)$ will be related to the dynamic transmission. For a TLL model, the same conductance results were found by the author by computing the current in response to an appropriate external electric field 9.10 More recently, they were confirmed by Blanter et a 11 through a self-consistent treatment of interactions, justified in the absence of backscattering. Other works based on the Kubo formula in a TLL with leads found different results due to a different electric field profile to which current is very sensitive. 12

An underlying hypothesis of Landauer's approach for noninteracting systems 1 is the ideal nature of the contacts, ensuring that emerging electrons are absorbed without reflection by the reservoirs. 1 Such a concept cannot be extended to interacting systems, as illustrated in Ref. [4.13]14 Rather, interactions give rise to collective excitations, or Laughlin quasiparticles in edge states, that are different from the electrons in the reservoirs. An emerging "ayasiparticle" undergoes a quasi-Andreev type reflection $19,10,15$ at a perfect contact with a reservoir.

This paper is mainly concerned with systems connected locally to reservoirs, such as quantum wires, or nanotubes; edge states couple differently to reservoirs, 14 and the present scattering approach will be extended elsewhere.16

Consider an arbitrary one-dimensional finite wire delimited by $[-a, a]$. The long wavelength part of the electronic density can be decpmposed into right and leftmoving electron densities $17 \rho_{+}$and $\rho_{-}$including implicitly the zero modes, $\rho=\rho_{+}+\rho_{-}$where spin is ignored for simplicity. For $r= \pm$, the boson field $\Phi_{r}$ defined by $\rho_{r}=-\partial_{x} \Phi_{r} / 2 \pi$ is the canonical conjugate to $r \rho_{r}$ (Kac-Moody algebra). The kinetic Hamiltonian is $H_{k i n}=\int_{-a}^{a} h v_{F}\left(\rho_{+}+\rho_{-}^{2}\right) / 2$. Any interaction Hamiltonian $H_{\text {int }}$ either between electrons or with impurities can be expressed as a functional of $\Phi_{+}, \Phi_{-}$, thus the total Hamiltonian,

$$
H=H_{\text {kin }}+H_{\text {int }}+e V_{\text {gate }} Q=H\left(\rho_{+}, \rho_{-}\right),
$$

is a functional of $\rho_{ \pm}$. Coupling to a gate is incorporated, and $Q=\int_{-a}^{a} \rho(x)$.

The current field $j(x)$ can be expressed independently of the dynamics, in or out-of-equilibrium. For this, the Hamiltonian $H^{(A)}$ in the presence of a vector potential $A$, is used :

$$
j(x)=-\left.\frac{\partial H^{(A)}}{\partial A(x)}\right|_{A=0} .
$$

$A$ can be absorbed by a gauge transformation of the right and left-going Fermion fields, $\Psi_{r} \sim e^{i r \Phi_{r}}$ for $r= \pm .17$ This is accomplished by the substitution

$$
\Phi_{r}(x) \rightarrow \Phi_{r}(x)-\frac{r e}{\hbar} \int^{x} A\left(x^{\prime}\right) d x^{\prime} .
$$

Taking the spatial derivative, one obtains $H^{(A)}$ as

$$
H^{(A)}=H\left(\rho_{+}+\frac{e}{h} A, \rho_{-}-\frac{e}{h} A\right) .
$$


Differentiating with respect to $A$ [Eq. (2)] yields 18

$$
j(x)=\frac{e}{h}\left[\mu_{+}(x)-\mu_{-}(x)\right] .
$$

For $r= \pm, \mu_{r}$ are operators that play a central role :

$$
\mu_{r}(x)=\frac{\partial H}{\partial \rho_{r}(x)}=h v_{F} \rho_{r}+\frac{\partial H_{i n t}}{\partial \rho_{r}(x)}+e V_{\text {gate }} .
$$

Also of use will be their sum,

$$
\mu(x)=\frac{\partial H}{\partial \rho(x)}=\frac{1}{2}\left[\mu_{+}(x)+\mu_{-}(x)\right] .
$$

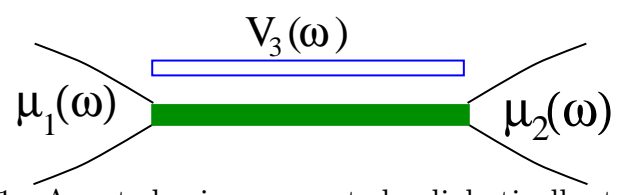

FIG. 1. A gated wire connected adiabatically to charge reservoirs with time-dependent electrochemical potential $\mu_{1,2}(t)=e^{i \omega t} \mu_{1,2}(\omega)$. The boundary conditions apply in the presence of arbitrary backscattering inside the wire.

Consider now a typical transport measurement, where one connects the wire adiabatically at $\pm a$ to charge reservoirs [see Figure]. Spatial and temporal structure on the scale of $\lambda_{F}$ (Friedel oscillations) are ignored. The local Hamiltonian density $\mathcal{H}( \pm a)$ is quadratic in $\rho_{ \pm}$when backscattering is irrelevant, so that $\mu_{ \pm}( \pm a)$ are linear in $\rho_{ \pm}$. One can think of the expectation value of $\mu_{+}(x)$ as the energy required to add a right-going electron at $x$. It includes the local Fermi energy $h v_{F} \rho_{+}(x)$, the interaction energy, and the gate potential that shifts the bottom band. In some sense, it is a local electro-chemical potential for right-going electrons. On the other hand, the left (right) reservoir injects bare right (left)-going electrons with a well defined electro-chemical potential $\mu_{1}\left(\mu_{2}\right)$. At the contacts where both incident fluxes impinge, the energy is conserved in the absence of any dissipation processes. Thus the field $\mu_{+}(-a, t)\left[\mu_{-}(a, t)\right]$ is required to be pinned to $\mu_{1}\left[\mu_{2}\right]$ at any time. To extend this conjecture to alternative regimes, time variation has to be slow enough so that the reservoirs are driven adiabatically through a sequence of equilibrium states with well defined time-dependent electro-chemical potential:

$$
\begin{aligned}
\mu_{+}(-a, t) & =\mu_{1}(t) \\
\mu_{-}(a, t) & =\mu_{2}(t) .
\end{aligned}
$$

Without interactions near the contacts, $\mu_{ \pm}(\mp a, t)=$ $h v_{F} \rho_{ \pm}(\mp a, t)+e V_{\text {gate }}(t)$; Eq. (6) imposes the density for incident electrons, and generalizes the Landauer concept to AC transport with arbitrary backscattering. But in the presence of interactions, $\mu_{ \pm}$depend on both $\rho_{+}$ and $\rho_{-}$, thus the density for incident electrons is not imposed, in contradiction with Ref.[19]. Rather, electrons are partially reflected, giving rise to a contact resistance. Indeed, Eq. (6) leads to a discontinuous local electrostatic potential $V_{l o c}$. $e V_{l o c}$ follows the electro-chemical potential on the reservoir side, while

$$
e V_{l o c}(x)=\frac{\partial\left[H-H_{k i n}\right]}{\partial \rho(x)}=\mu(x)-\frac{h v_{F}}{2} \rho(x)
$$

on the interacting side. 10 Note that $e V_{\text {gate }}$ is included. $\mu(x)$ is a local electro-chemical potential for both carriers [Eq. (5)]. This clarifies a confusing point in the self-consistent treatment of interactions in higherdimensional systems, where the continuity of $V_{l o c}$ is expected, even though not implemented in the results. It would be interesting to define and then verify analogous conditions to Eq. (6).

The AC conductance matrix $\mathbf{G}_{\mathbf{3}}(\omega)$ will be now expressed formally, where $\omega$ is the external frequency. In the sequel, I switch to the Fourier transform of expectation values in a time-dependent ground state. $\mathbf{G}_{\mathbf{3}}(\omega)$ is a $3 \times 3$ matrix with $g_{\alpha \beta}=\delta I_{\alpha} / \delta V_{\beta}$ where $V_{1,2}=\mu_{1,2} / e$, $V_{3}=V_{\text {gate }}, I_{1,2}=\mp j(\mp a, \omega)$ and $I_{3}$ the displacement current. A complete description with all the surrounding three-dimensional environment would be too complex. Instead, assume that all the electric field lines emerging from the wire end up on the gate, thus the latter carries an opposite charge to that on the wire $Q(\omega)$. This ensures Kirchoff's law, $\sum_{\alpha} I_{\alpha}=0$ because, using the continuity equation,

$$
\begin{aligned}
I_{3}(\omega) & =-i e \omega Q(\omega)=\int_{-a}^{a} i \omega e \rho(x, \omega) \\
& =-\int_{-a}^{a} \partial_{x} j(x, \omega)=-I_{1}(\omega)-I_{2}(\omega) .
\end{aligned}
$$

On the other hand, $V_{\text {gate }}(\omega)$ appears as a reference potential in Eqs. (4,6). Thus the two constraints on the conductance matrixa

$$
\sum_{\alpha} g_{\alpha \beta}=0=\sum_{\beta} g_{\alpha \beta},
$$

are ensured. Next focus on the first $2 \times 2$ block of $\mathbf{G}_{\mathbf{3}}(\omega)$ denoted $\mathbf{G}_{\mathbf{2}}(\omega)$. Using Eqs. (6.63),

$$
\begin{gathered}
I_{1}=-j(-a, \omega)=\frac{e}{h}\left[\mu_{-}(-a, \omega)-\mu_{1}(\omega)\right], \\
I_{2}=j(+a, \omega)=\frac{e}{h}\left[\mu_{+}(-a, \omega)-\mu_{2}(\omega)\right] .
\end{gathered}
$$

In order to express $I_{\alpha}$ to linear order in $\mu_{1,2}=$ $\mu_{ \pm}(\mp a, \omega)$, it is sufficient to retain the linear dependence of $\mu_{ \pm}( \pm a, \omega)$, determined by some matrix $\mathbf{S}(\omega)$,

$$
\left(\begin{array}{c}
\mu_{-}(-a, \omega) \\
\mu_{+}(a, \omega)
\end{array}\right)=\mathbf{S}(\omega)\left(\begin{array}{c}
\mu_{+}(-a, \omega) \\
\mu_{-}(a, \omega)
\end{array}\right) .
$$

Combined with Eq. (10), this gives

$$
\mathbf{G}_{\mathbf{2}}(\omega)=\frac{e^{2}}{h}[\mathbf{S}(\omega)-\mathbf{I}]
$$


In order to interpret $\mathbf{S}(\omega)$ as a "scattering" matrix, one can model the transition region by an $\mathrm{N}$-channel system where electrons are injected at the same quasiequilibrium distribution. On the reservoir side of $-a$, the total current is $J_{+}(\omega)-J_{-}(\omega)$, where $J_{+}(\omega)=$ $N e \mu_{1}(\omega) / h$ is the incident current, and $J_{-}(\omega)$ the unknown reflected current. The local continuity of the current at $-a$ together with Eq. (3) imply that $e \mu_{-}(-a, \omega) / h=J_{-}(\omega) / N$ is the average reflected current per channel. Similarly, $e \mu_{+}(a, \omega) / h$ is the average transmitted current to the right reservoir. If the elements of $\mathbf{S}(\omega)$ are denoted as follows

$$
\mathbf{S}(\omega)=\left(\begin{array}{ll}
R(\omega) & T^{\prime}(\omega) \\
T(\omega) & R^{\prime}(\omega)
\end{array}\right)
$$

then $T(\omega)[R(\omega)]$ can be viewed as the total dynamic transmission [reflection] coefficient for the incident flux from the left to the right reservoir [into the left reservoir]. $T^{\prime}$ and $R^{\prime}$ play the same role for the right reservoir. Nevertheless, important differences from the usual scattering approach should be stressed. The elements of $\mathbf{S}(\omega)$ determine directly the current or density, but are nonetheless complex numbers. $\mathbf{S}(\omega)$ is not unitary, and in general not symmetric unless there is a perfect reflection symmetry. In addition, $T(\omega)+R(\omega) \neq 1$, and current conservation is ensured by the gate [Eq. (8)]. Finally, using Eq. (12), and letting the $\omega$ dependence be implicit :

$$
\mathbf{G}_{3}=\frac{e^{2}}{h}\left(\begin{array}{ccc}
R-1 & T^{\prime} & 1-R-T^{\prime} \\
T & R^{\prime}-1 & 1-R^{\prime}-T \\
1-R-T & 1-R^{\prime}-T^{\prime} & R+R^{\prime}+T+T^{\prime}-2
\end{array}\right)
$$

At zero frequency, $S(0)$ becomes real symmetric, and $T(0)+R(0)=T^{\prime}(0)+R^{\prime}(0)=1$, but $R(0)$ can be negative. Then the DC conductance is

$$
g=g_{12}=-g_{11}=T(0) \frac{e^{2}}{h} .
$$

As a first application of these boundary conditions, consider now a model where both total charges $Q_{ \pm}=$ $\int_{-a}^{a} \rho_{ \pm}(x) d x$ are conserved,

$$
\left[Q_{ \pm}, H\right]=0
$$

Then it is shown here that $T(0)=1$. For a quadratic Hamiltonian, this result was shown in Refs.[ 4.7], and justifies the hypothesis adopted in Refs. [6].

In the Heisenberg representation, an operator $O$ evolves according to $i \hbar d O / d t=[H, O]+i \hbar \partial O / \partial t$, but $\partial O / \partial t=0$ in the stationary regime. For $r= \pm, \Phi_{r}$ is the canonical conjugate to $\rho_{r}$, thus $d \Phi_{r} / d t=-r \mu_{r} / \hbar[$ Eq. (州]. Then $h d \rho_{r} / d t=r \partial_{x} \mu_{r}$ is an equation for field operators that one can integrate between $-a$ and $a$ to get, using Eq. (16),

$$
\mu_{r}(a, t)-\mu_{r}(-a, t)=r h \frac{d Q_{r}}{d t}=0
$$

On the other hand, the field $\mu_{+}(-a, t)$ cannot fluctuate but is equal to $\mu_{1}$ [Eq. (6)], thus $\mu_{+}(a, t)=\mu_{1}$ at all times. Similarly, $\mu_{-}(-a, t)=\mu_{-}(a, t)=\mu_{2}$. Thus $T(0)=1$ and $R(0)=0$ [see Eq. (11)], and the DC conductance is equal to $e^{2} / h$ [see Eq. (15) or simply Eq. (3)].

A second application is to investigate ac transport in the simplest model (verifying Eq. (16)) : the TLL model. The matrix $\mathbf{S}(\omega)$ in Eq. (11) can be computed in an instructive way, through a "transfer" matrix $\mathbf{A}(\omega)$ such that

$$
\boldsymbol{\mu}(a, \omega)=\mathbf{A}(\omega) \boldsymbol{\mu}(-a, \omega)
$$

where $\boldsymbol{\mu}$ stands for the vector $\left(\mu_{+}, \mu_{-}\right)$. For this, it is convenient to use the right- and left-propagating current modes $j_{ \pm}$, corresponding to up and down edgeexcitations in a Hall bar,20 that can be denoted "quasiparticles". The Hamiltonian density is given by $\mathcal{H}(x)=$ $h\left(j_{+}^{2}+j_{-}^{2}\right) / 2 e^{2} u K$, where $u$ and $K$ are interaction parameters 17 Without interactions, $j_{ \pm}=e v_{F} \rho_{ \pm}, u=v_{F}$ and $K=1 . j_{ \pm}$propagate freely at the sound velocity $u$, thus

$$
\mathbf{j}(a, \omega)=e^{i \boldsymbol{\sigma}_{\boldsymbol{z}} \omega t_{L}} \mathbf{j}(-a, \omega),
$$

where $t_{L}=2 a / u$ is the transit time of the wire and $\boldsymbol{\sigma}_{\boldsymbol{z}}$ the $\mathrm{z}$ Pauli matrix. On the other hand, $j_{ \pm}$are related to $\rho_{ \pm}$by simple diagonalization, but their relation to $\mu_{ \pm}$is of more use here :

$$
\begin{aligned}
\mathbf{j}(x, \omega) & =\frac{e}{h} \mathbf{M} \boldsymbol{\mu}(x, \omega) \\
\mathbf{M} & =\frac{1}{1+\gamma}\left(\begin{array}{cc}
1 & -\gamma \\
-\gamma & 1
\end{array}\right),
\end{aligned}
$$

where the coefficient $\gamma$ is given by

$$
\gamma=\frac{1-K}{1+K}
$$

Then $\mathbf{M}^{-1}$ can be obtained from $\mathbf{M}$ by $\gamma \rightarrow-\gamma$. Equations (20,19) yield the "transfer" matrix

$$
\mathbf{A}(\omega)=\mathbf{M}^{-1} e^{i \boldsymbol{\sigma}_{\boldsymbol{z}} \omega t_{L}} \mathbf{M} .
$$

This allows to deduce the scattering matrix $\mathbf{S}(\omega)$ in Eq. (11), symmetric due to the reflection symmetry,

$$
\begin{aligned}
& T(\omega)=T^{\prime}(\omega)=(1-\gamma) \frac{e^{-i \omega t_{L}}+\gamma e^{i \omega t_{L}}}{e^{-i \omega t_{L}}-\gamma^{2} e^{i \omega t_{L}}}, \\
& R(\omega)=R^{\prime}(\omega)=\gamma\left[1-e^{i \omega t_{L}} T(\omega)\right] .
\end{aligned}
$$

One can show that $|\operatorname{Det} \mathbf{S}(\omega)|=1$, which is a constraint that has to hold for any quadratic Hamiltonian with timereversal symmetry. Note that $\mathbf{S}(\omega)$ depends solely on the intrinsic properties of the TLL model; the boundary conditions (6) allow to express the AC conductance matrix through $\mathbf{S}(\omega)$, Eq. (14). I now analyze in more details the capacitive effects. The gate conductance is 


$$
g_{33}(\omega)=2 \frac{e^{2}}{h}(1-\gamma) \frac{1-e^{i \omega t_{L}}}{1+\gamma e^{i \omega t_{L}}} .
$$

Thus the "electro-chemical" capacitance of the wire per unit length with respect to the gate 10.11

$$
C=-\lim _{\omega \rightarrow 0}\left[\frac{g_{33}(\omega)}{i \omega 2 a}\right]=2 \frac{K}{u} \frac{e^{2}}{h},
$$

is proportional to the compressibility. 17 This result can be checked by minimizing the zero mode contribution to $H$ at fixed total current, thus by minimizing $h u Q^{2} / 8 a K+$ $e Q V_{\text {gate }}$. $C$ results from two capacitors in series: its value without interactions $C_{0}=e^{2} d n / d E=e^{2} h v_{F} / 2$, of purely kinetic origin, and the "electrostatic" capacitance $c$, obtained by evaluating Eq. (7), 2, 21, 11

$$
\begin{aligned}
c=e \frac{\delta \rho}{\delta V_{l o c}} & =\frac{e^{2}}{h}\left(\frac{u}{K}-v_{F}\right)^{-1} . \\
\frac{1}{C} & =\frac{1}{c}+\frac{1}{C_{0}} .
\end{aligned}
$$

Interestingly, evaluating then differentiating Eqs. (7.5) with respect to $\rho$ allows to recover Eq. (27). In the TLL $\mu(x)=e^{2} \rho(x) / C+e V_{\text {gate }}$ justifying its interpretation as a local electrochemical potential for both carriers in Ref.[ 7]. But measuring $C$ gives the ratio $K / u$, leaving both $u$ and $K$ unknown. For usual ballistic quantum wires, where $a$ is several $\mu m, 2 \pi / t_{L} \sim G H z$ is quite high. One is often in the regime $\omega t_{L} \ll 1$, where the non-dissipative part10,11] of $g_{33}(\omega)$ [Eq. (25)] is $-\operatorname{Im}\left[g_{33}(\omega)\right] \simeq 2 X-$ $X^{3}\left(1-1 / 3 K^{2}\right) / 2$, with $X=C a \omega$. A strategy consists in measuring the leading term $2 X$, then the subleading term that one divides by $X^{3} / 2$ to infer $1-1 / 3 K^{2}$, thus $K$. $u$ can be then determined from $C$.

The underlying dynamics are now interpreted. Eq. (20) is equivalent to

$$
\left(\begin{array}{c}
e \mu_{-}(x, \omega) / h \\
j_{+}(x, \omega)
\end{array}\right)=\left(\begin{array}{cc}
\gamma & 1+\gamma \\
1-\gamma & -\gamma
\end{array}\right)\left(\begin{array}{c}
e \mu_{+}(x, \omega) / h \\
j_{-}(x, \omega)
\end{array}\right)
$$

so that the matrix on the right hand side can be viewed as a local "scattering" matrix. 19 Let us focus for instance on $x=-a$ where $\mu_{+}(-a, \omega)=\mu_{1}(\omega)$. When no charge is incident from the left reservoir, i.e. $\mu_{1}=0$, then $j_{+}=-\gamma j_{-} ;-\gamma$ is the reflection coefficient for a "quasiparticle" incident on the contact. For repulsive interactions, $K<1$, thus $-\gamma<0$; a "quasihple" is reflected, in analogy with Andreev reflection, 4, 9. 10.15 If no "quasiparticle" comes from the right, i.e. $j_{-}=0$, then one finds

$$
j_{+}=\frac{e}{h}(1-\gamma) \mu_{1}
$$

and thus $K_{a}=2 K /(1+K)=1-\gamma$ is the transmission coefficient for the incident flux from the reservoir. $T(\omega)$ and $R(\omega)$ result from the multiple reflections on the contacts, in analogy with a Fabry-Perot resonator. 422 They have resonances at the collective modes of the finite wire $\omega_{n}=u q$ for $q=2 n \pi / 2 a$, at which $T=1$ and $R=0$. This is because $e^{i \omega_{n} \boldsymbol{\sigma}_{\boldsymbol{z}} t_{L}}=\mathbf{I}$, thus Eq. (22) becomes $\mathbf{A}\left(\omega_{n}\right)=\mathbf{M} \mathbf{M}^{-1}=\mathbf{I} 10$ Note that since $j=j_{+}-j_{-}$, Eq. (29) obtained for $j_{-}=0$ yields the current at the interface of a semi-infinite TLL and a Fermi liquid, the DC conductance becomes $g_{a}=K_{a} e^{2} / h$. 4.96

Indeed, all the above scattering matrices have been encountered in Refs.[ [, 9, 10], where a TLL is connected perfectly to noninteracting leads at $\pm a$.

More generally, consider the case where $H_{\text {int }}$ is an arbitrary quadratic functional of $\rho$, vanishing for $|x|>a$. If an electron impinges at $t=0$ on $-a$, i.e. $\rho_{+}(x, t=$ $0)=\delta(x+a)$, the transmitted [respectively reflected] charge to $a[$ at $-a]$ at time $t$, i.e. $\rho_{+}(a, t)\left[\rho_{-}(-a, t)\right]$ is given by the function $M_{++}(a,-a, t)\left[M_{-+}(-a,-a, t)\right]$ whose Fourier transform coincide exactly with $T(\omega)$ (respectively $R(\omega))$ in Eq. (28). In addition, these functions determine the non local dynamic conductivity at the contacts, 19

$$
\begin{aligned}
\sigma(a,-a, \omega) & =\frac{e^{2}}{h} T(\omega) \\
\sigma(a, a, \omega) & =\frac{e^{2}}{h}[1-R(\omega)] .
\end{aligned}
$$

The reservoirs can be modeled by an external potential that drops only at the contacts, 10 so that, taking into account the constant gate potential in $[-a, a]$,

$$
E(x, \omega)=\left[V_{1}-V_{\text {gate }}\right] \delta(x+a)-\left[V_{2}-V_{\text {gate }}\right] \delta(x-a) .
$$

Then $j( \pm a, \omega)=\sigma(a, \mp a, \omega)\left[V_{1,2}(\omega)-V_{\text {gate }}(\omega)\right]$, leading to the same $\mathbf{G}_{\mathbf{3}}(\omega)$ [Eq. (14)] by use of Eqs. (30,8). 1, 10]

This is not a pure coincidence. The action being quadratic, the ground state properties are given exactly by minimizing it. The equation of motion thus obtained imposes the continuity 4 of both $j=e\left[\mu_{+}-\mu_{-}\right] / h$ and that of Eq. (5), equals for instance $\mu(x)=h u \rho / K+$ $e V_{\text {gate }}$ in the TLL. Here the $\omega$ dependence is implicit. Thus both $\mu_{+}$and $\mu_{-}$are continuous. For any $|x|>a$, $\mu_{ \pm}(x)=h v_{F} \rho_{ \pm}(x)$ (see Eq. (4)). The left reservoir injects electrons with density $\rho_{+}\left(-a^{(-)}, \omega\right)=\mu_{1}(\omega) / h v_{F}$ on the noninteracting lead side, thus $\mu_{+}\left(-a^{(-)}, \omega\right)=$ $\mu_{1}(\omega)$. This fixes the continuous field $\mu_{+}$on the interacting side, $\mu_{+}\left(-a^{(+)}, \omega\right)=\mu_{1}$, which is exactly Eq. (6). Similar reasoning holds symmetrically for the right reservoir. Also $\mu_{-} / h$ has to be continuous at $-a$, thus it is equal to $v_{F} \rho_{-}$, the reflected current. This is in accordance with the previous general interpretation of $\mathbf{S}(\varphi)$ since one-dimensional leads model an effective channel.14

These arguments in favor of the noninteracting leads are restricted to a quadratic density Hamiltonian $\mathcal{H}(x)$ along the wire, although some of them might be extended if only $\mathcal{H}( \pm a)$ is quadratic. The role of backscattering in a TLL connected to leads 9310 was found to be controlled by the Fabry-Perot dynamics recovered here by using Eq. (6), but the equivalence has to be checked 
and might be limited to linear transport. The boundary conditions (6), formulated without connecting leads, are more general, and offer possibilities for future studies. One has to reformulate the bosonisation procedure to compute the correlation functions. Implementing Eq. (6) in a path integral formalism would give access to the nonlinear regime, AC transport, and current fluctuations. Conceptually, the scattering approach presented here can be extended to situations where linear response theory fails, as will be the subject of a future study on edge states and many channel systems. 16

Acknowledgments : I am indebted to T. Martin for reading the manuscript and for useful criticism. I would like to acknowledge fruitful discussions with A. Alekseev, Y. Blanter, D. Bernard, D. C. Glattli, T. Jolicoeur, and H. J. Schulz.

Note : In a recent erratum 24 Egger and Grapert modified their boundary conditions for the TLL 19 by using self-consistent arguments.21 Their corrected results agree with Ref. [ 4] and therefore with its present generalization.

${ }^{1}$ R. Landauer, Phil. Mag. 21, 863 (1970); Y. Imry, R. Landauer, and S. Pinhas, Phys. Rev. B 31, 6207 (1985).

${ }^{2}$ M. Büttiker, A. Prêtre, and H. Thomas, Phys. Rev. Lett. 70, 4114 (1993).

${ }^{3}$ N. S. Wingreen, A. P. Jauho, and Y. Meir, Phys. Rev. B 48, R8487 (1993-I); C. Bruder and H. Scholler, Phys. Rev. Lett. 72, 1076 (1994).

${ }^{4}$ I. Safi and H. J. Schulz, Phys. Rev. B 52, R17040 (1995).

${ }^{5}$ D. Maslov and M. Stone, Phys. Rev. B 52, R5539 (1995);

V. V. Ponomarenko, ibid R8666.

${ }^{6}$ A. Y. Alekseev, V. Cheianov, and J. Fröhlich, Phys. Rev. B 54, R17320 (1996); A. Shimizu, J. Phys. Soc. Jpn. 65, 1162 (1996); The first argument by Y. Oreg and A. A Finkel'stein, Phys. Rev. B 54, R14265 (1996) is similar,22 but is different from the second argument proposed also by A. Kawabata, J. Phys. Soc. Jpn. 65, 30 (1996), and based on Kubo formula withgit reservoirs; this yields in general a different conductanced.

${ }^{7}$ I. Safi, Phys. Rev. B 55, R7331 (1997-II).

${ }^{8}$ A. Alekseev, V. Cheianov and J. Fröhlich, condmat/9803346.

${ }^{9}$ I. Safi and H. J. Schulz, in Quantum Transport in Semiconductor Submicron Structures, edited by B. Kramer (Kluwer Academic Press, Dordrecht, 1995); in Correlated Fermions and Transport in Mesoscopic Systems, edited by T. Martin, G. Montambaux, and J. T. Van (Editions Frontieres, Gif-sur-Yvette, 1996); Phys. Rev. B, 58, August 1998 (in press), cond-mat/9803326.

10 Inès Safi-Taktak, Ph.D. thesis, Laboratoire de Physique des Solides, 1996; Ann. Phys. (Paris) 22, 463 (1997).

${ }^{11}$ Ya. M. Blanter, F. W. J. Hekking et M. Büttiker, condmat/9710299.
${ }^{12}$ V. V. Ponomarenko, Phys. Rev. B 52, R8666 (1995); V. A. Sablikov and B. S. Shchamkhalova, JETP Lett 66, 41 (1997).

${ }^{13}$ C. L. Kane and M. P. A. Fisher, Phys. Rev. B 52, 17393 (1995).

${ }^{14}$ C. de C. Chamon and E. Fradkin, Phys. Rev. B 55, 4534 (1997-II).

15 N. P. Sandler, C. de C. Chamon, and E. Fradkin, Phys. Rev. B 57, 12324 (1998).

${ }^{16}$ Inès Safi, in preparation.

17 S. Tomonaga, Prog. Theor. Phys. 5, 544 (1950); D. C. Mattis and E. H. Lieb, J. Math. Phys. 6, 304 (1965).

${ }^{18}$ For the specific case of a quadratic Hamiltonian, and in equilibrium, see also Ref.[22].

${ }^{19}$ R. Egger and H. Grabert, Phys. Rev. Lett. 77, 538 (1996).

${ }^{20}$ X. G. Wen, Phys. Rev. Lett. 64, 2206 (1990).

${ }^{21}$ R. Egger and H. Grabert, Phys. Rev. Lett. 79, 3463 (1997).

${ }^{22}$ Y. Oreg and A. M. Finkel'stein, Phys. Rev. Lett. 74, 3668 (1993).

23 A. Furusaki and N. Nagaosa, Phys. Rev. B 54, R5239 (1996). See also D. Maslov, Phys. Rev. B 52, R44368 (1995), but where results are restricted to the bulk 10 .

${ }^{24}$ R. Egger and H. Grabert, Phys. Rev. Lett. 80, 2255 (1998). 